${ }^{6}$ E. C. Bower, "Coefficients for interpolating a function directly from a table of double integration." Lick Observatory, Bull., no. 445 , v. 16, 1932, p. 42.

${ }^{7}$ Interpolation and Allied Tables, reprinted from the British Nautical Almanac for 1937, 4 th edition with additions. (London, H. M. Stationery Office, 1946.)

${ }^{8}$ W. E. Milne, Numerical Calculus. Princeton, 1949.

${ }^{9}$ L. Fox \& E. T. GoodwIN, "Some new methods for the numerical integration of ordinary differential equations," MS. awaiting publication.

\title{
Formulas for Calculating the Error Function of a Complex Variable
}

1. Various methods have been suggested for the computation, to high accuracy, of the error function $\Phi(Z)=\int_{0}^{z} e^{-u^{2}} d u$ for complex arguments $Z=X+i Y$. The Maclaurin series is convenient for extreme accuracy only when $|Z|$ is small, and the asymptotic expansions ${ }^{1}$ are useful only for fairly large values of $|Z|$. Other less elementary methods (e.g., the AIREy converging factor, ${ }^{2}$ or the continued fraction expansion ${ }^{1}$ ) have limited success in certain regions of the $Z$-plane. The most convenient methods for calculating $\Phi(Z)$ to very many figures are described in the works of MILLER \& GORDON $^{3}$ and ROSSER. ${ }^{4}$ It is the purpose of this note, which is self-contained, to present in a concise and practical form, two schemes for the computation of $\Phi(Z)$ which follow the main ideas of Miller, Gordon, and Rosser.

2. The basis of the present methods is the following formula:

$$
\sum_{n=-\infty}^{\infty} \exp \left(-(u+n a)^{2}\right)=\pi^{\frac{3}{3}} a^{-1} \sum_{n=-\infty}^{\infty} \exp \left(-n^{2} \pi^{2} a^{-2}\right) \cos (2 n \pi u / a)
$$

which is an immediate corollary of Porsson's formula. ${ }^{5}$ Formula (1) is also essentially Jacobi's imaginary transformation, ${ }^{6}$ which has long been familiar to a number of mathematicians and physicists. ${ }^{7,8}$ Also, (1) was used by DAwsoN $^{9}$ in his computation of $\int_{0}^{Y} e^{u^{2}} d u$. Two different methods for calculating $\Phi(Z)$ are given here in $\mathbf{4}$ and $\mathbf{5}$. In all formulas, summations are from 1 to $\infty$ except where otherwise noted. The symbol $\doteq$ will denote approximate equality.

3. Dawson's formula for $\int_{0}^{Y} e^{u^{2}} d u$ is needed in the second method. It follows from the approximate equality obtained from (1), which is

$$
e^{u^{2}}(1+E)=a \pi^{-\frac{1}{2}}\left(1+2 \sum \exp \left(-a^{2} n^{2}\right) \cosh 2 n a u\right), \quad a \leqslant 1
$$

where the relative error $E=2 \sum e^{-n^{2} \pi^{2} / a^{2}} \cos (2 n \pi u / a)$, is of the order of magnitude of $2 e^{-\pi^{2} / a^{2}}$, since $2 e^{-4 \pi^{2} / a^{2}}$ is very small by comparison. Approximate values of $E$ are given in the following table:

$$
\begin{array}{ccccccc}
a & 1 & 0.9 & 0.8 & 0.7 & 0.6 & 0.5 \\
\hline E & 10^{-4} & 10^{-5} & \frac{1}{2} \cdot 10^{-6} & \frac{1}{2} \cdot 10^{-8} & \frac{1}{4} \cdot 10^{-11} & \frac{3}{2} \cdot 10^{-17}
\end{array}
$$

Putting $a=\frac{1}{2}$ in (2) Dawson obtains

$$
e^{u^{2}} \doteq \pi^{-\frac{1}{2}}\left(\frac{1}{2}+\sum e^{-n^{2} / 4} \cosh n u\right),
$$

the relative error in (3) being less than $2 \cdot 10^{-17}$. Then integration of (3) results in

$$
\int_{0}^{Y} e^{u^{2}} d u \doteq \pi^{-\frac{1}{2}}\left(\frac{1}{2} Y+\sum n^{-1} \exp \left(-n^{2} / 4\right) \sinh n Y\right) .
$$


4. One method of obtaining a formula for $\Phi(Z)$ is to choose the path of integration from 0 to $Z$ to consist of the line segments $[0, X]$ and $[X, X+i Y]$. We find

(5) $\Phi(Z)=\int_{0}^{X} e^{-u^{2}} d u+e^{-X^{2}} \int_{0}^{Y} e^{u^{2}} \sin 2 X u d u$

$$
+i e^{-X^{2}} \int_{0}^{Y} e^{u^{2}} \cos 2 X u d u
$$

The first term on the right is numerically well known. ${ }^{10}$ To deal with the other terms, we multiply across in (3) by $\sin 2 X u$ and $\cos 2 X u$ respectively, and integrate between 0 and $Y$. Using the explicit expressions for integrals of the form $\int \cosh n u\left\{\begin{array}{l}\sin q u \\ \cos q u\end{array}\right\} d u$ we readily obtain $\Phi(Z)=A+i B$, where

(6) $A \doteq \int_{0}^{X} e^{-u^{2}} d u+\pi^{-\frac{1}{2}} e^{-X^{2}}(1-\cos 2 X Y) / 4 X$ $+\pi^{-\frac{1}{3}} e^{-X^{2}} \sum\left(n^{2}+4 X^{2}\right)^{-1}(2 X-2 X \cosh n Y \cos 2 X Y$

$+n \sinh n Y \sin 2 X Y) \cdot \exp \left(-n^{2} / 4\right)$

and

$$
\begin{aligned}
B=\pi^{-1} e^{-X^{2}}\left((4 X)^{-1} \sin 2 X Y+\sum\left(n^{2}+4 X^{2}\right)^{-1} \exp \left(-n^{2} / 4\right)\right. \\
\cdot\{2 X \cosh n Y \sin 2 X Y+n \sinh n Y \cos 2 X Y\}) .
\end{aligned}
$$

5. Another formula for calculating $\Phi(Z)$ is found by choosing the path of integration to consist of the line segments $[0, i Y]$ and $[i Y, X+i Y]$, when we find

$$
\begin{aligned}
\Phi(Z)=i \int_{0}^{Y} e^{u^{2}} d u+e^{Y^{2}} \int_{0}^{X} e^{-u^{2}} \cos 2 Y u d u & \\
& -i e^{Y^{2}} \int_{0}^{X} e^{-u^{2}} \sin 2 Y u d u .
\end{aligned}
$$

The first term on the right can be found from Dawson's approximation in (4). The other two terms are found by letting $a=12$ in (1), then multiplying across by $\cos 2 Y u$ and $\sin 2 Y u$, and integrating between 0 and $X$. In view of the fact that $\int_{6}^{\infty} e^{-u^{2}} d u$ is negligible, being of order $10^{-17}$, it is easily shown that if we neglect the integral of everything arising from $n \neq 0$ in the left member of (1), when $X \leqslant 6$ the error is $<2 \int_{6}^{\infty} e^{-u^{2}} d u$. When $X>6$, we can replace $X$ by 6 in (8), with a relative error $<\int_{6}^{\infty} e^{-u^{2}} d u$, and in view of the result in the preceding sentence, the total error is surely $<3 \int_{6}^{\infty} e^{-u^{2}} d u$. For maximum accuracy it is not necessary to take more than 24 terms arising from under the summation sign in the right member of (1), because then the truncating error, which is of the order of $e^{-4 \pi^{2}}$, is less than the error in the approximation formula. Making use of the explicit expressions for integrals of the form $\int \cos p u\left\{\begin{array}{c}\sin q u \\ \cos q u\end{array}\right\} d u$, whenever $|Y| \neq n \pi / 12$, we obtain $\Phi(Z)=C+i D$, where

(9) $C \doteq \frac{\pi^{\frac{1}{2}}}{12} e^{Y^{2}}\left((2 Y)^{-1} \sin 2 X Y+72 \sum_{n=1}^{24}\left(n^{2} \pi^{2}-144 Y^{2}\right)^{-1} \exp \left(-n^{2} \pi^{2} / 144\right)\right.$ $\left.\cdot\left\{\frac{1}{6} \pi n \sin \frac{1}{6} \pi n X \cos 2 X Y-2 Y \cos \frac{1}{6} \pi n X \sin 2 X Y\right\}\right)$ 
and

$$
\begin{aligned}
D \doteq & \pi^{-\frac{1}{2}}\left(\frac{1}{2} Y+\sum n^{-1} \exp \left(-n^{2} / 4\right) \sinh n Y\right) \\
& -\frac{1}{12} \pi^{\frac{1}{2}} e^{Y^{2}}\left((2 Y)^{-1}(1-\cos 2 X Y)+72 \sum_{n=1}^{24}\left(n^{2} \pi^{2}-144 Y^{2}\right)^{-1}\right. \\
& \times \exp \left(-n^{2} \pi^{2} / 144\right)\left\{\frac{n \pi}{6} \sin \frac{1}{6} n \pi X \sin 2 X Y\right. \\
& \left.\left.\quad+2 Y \cos \frac{1}{6} \pi n X \cos 2 X Y-2 Y\right\}\right) .
\end{aligned}
$$

In the exceptional case when $|Y|=n \pi / 12$ for some value of $n$, omit that term from inside the summation signs in $C$ and $D$ and instead add

$$
\pi^{\frac{1}{3}}(48 Y)^{-1}(4 X Y+\sin 4 X Y-i\{1-\cos 4 X Y\}) .
$$

Both methods which are described in $\mathbf{4}$ and $\mathbf{5}$ are accurate to within a relative error of about $10^{-16}$ in $|\Phi(Z)|$, except for values of $|Y|$ very close to $n \pi / 12$ in the latter method. The amount of computational work seems to be about the same for these two methods, and in general it appears difficult to recommend one of the pairs of formulas (6), (7) or (9), (10) in preference to the other. Each method affords a good computational check upon the use of the other.

6. Often the value of $e^{Z^{2}} \Phi(Z)$ is desired because it is a smoother function which can be tabulated to a constant number of decimals. Thus from (6) and (7) we have at once $e^{Z^{2}} \Phi(Z)=E+i F$, where

$$
\begin{aligned}
E \doteq \exp & \left(X^{2}-Y^{2}\right) \cos 2 X Y \int_{0}^{X} e^{-u^{2}} d u \\
& +\pi^{-\frac{1}{2}} e^{-Y^{2}}\left((4 X)^{-1}(\cos 2 X Y-1)\right. \\
& \left.+2 X \sum\left(n^{2}+4 X^{2}\right)^{-1} \exp \left(-n^{2} / 4\right)\{\cos 2 X Y-\cosh n Y\}\right)
\end{aligned}
$$

and

$$
\begin{array}{r}
F \doteq \exp \left(X^{2}-Y^{2}\right) \sin 2 X Y \int_{0}^{X} e^{-u^{2}} d u+\pi^{-\frac{1}{2}} e^{-Y^{2}}\left\{(4 X)^{-1} \sin 2 X Y\right. \\
\left.+\sum\left(n^{2}+4 X^{2}\right)^{-1} \exp \left(-n^{2} / 4\right)(2 X \sin 2 X Y+n \sinh n Y)\right\}
\end{array}
$$

NBSCL

H. E. SAlzer

This work was sponsored in part by the Office of Air Research, AMC, USAF.

$1 \mathrm{~J}$. BuRgess, "On the definite integral $\frac{2}{\sqrt{\pi}} \int_{0}^{t} e^{-t^{2}} d t$ with extended tables of values,"

R. Soc. Edinburgh, Trans., v. 39, part II, 1898, p. 257-321.

2 J. R. AIREy, "The 'converging factor' in asymptotic series and the calculation of Bessel, Laguerre and other functions," Phil. Mag., s. 7, v. 24, 1937, p. 521-552.

3 W. L. Miller \& A. R. Gordon, "Numerical evaluation of infinite series," Jn. Phys. Chem., v. 35, 1931, especially part V, p. 2856-2857, 2860-2865.

${ }_{4} \mathrm{~J}$. B. Rosser, Theory and Application of $\int_{0}^{z} e^{-x^{2}} d x$ and $\int_{0}^{z} e^{-p^{2} y^{2}} d y \int_{0}^{y} e^{-x^{2}} d x$. Part I. Methods of Computation, New York, 1948.

${ }^{5} \mathrm{E}$. C. TITchmaRSH, Introduction to the Theory of Fourier Integrals. Oxford, 1937, p. 60-64.

${ }^{6}$ E. T. Whittaker \& G. N. Watson, A Course of Modern Analysis. Fourth ed., Cambridge, 1940, p. 124, 474-476.

${ }^{7}$ E. T. Goodwin, "The evaluation of integrals of the form $\mathcal{S}_{-\infty}{ }^{\infty} f(x) e^{-x^{2}} d x$," Cambridge Phil. Soc., Proc., v. 45, 1949, p. 241-245. 
8 A. M. Turing, "A method for the calculation of the Zeta-function," London Math. Soc., Proc., s. 2, v. 48, 1943, p. 180-197.

${ }^{9}$ H. G. DAwson, "On the numerical value of $\int_{0}^{h} e^{x^{2}} d x$," London Math. Soc., Proc., s. 1, v. 29,1898 , p. $519-522$.

10 NBS, Tables of Probability Functions. V. 1, New York, 1941.

\section{RECENT MATHEMATICAL TABLES}

857[D].-J. K. LyNCH, "Calculation of $(\sin x) / x$ tables," PostmasterGeneral's Department, Research Laboratory Report No. 3238, Melbourne, C. 1., (April 1949), C.E. 615/R, Case no. 2266. 7 mimeographed pages + drawing.

These tables of $(\sin x) / x$ were computed to facilitate the calculation of the harmonic components of periodic impulses. The function $(\sin x) / x$ is tabulated for $x=0\left(1^{\circ}\right) 180^{\circ}\left(5^{\circ}\right) 1800^{\circ} ; 4 \mathrm{D}$, with first differences. The drawing contains a graph of $(\sin x) / x$ for $x$ ranging from $0^{\circ}$ to $1600^{\circ}$, together with a straight line converting degrees to radians. The statement in the Introduction ". . . the interval of tabulations is such that linear interpolation will suffice," is not quite correct, since second differences are needed for full accuracy when $x$ lies between $180^{\circ}$ and $295^{\circ}$.

For another table of this function, see $[M T A C$, v. 4, p. 80].

H. E. Salzer

NBSCL

858[F, G, O].- R. E. BEARD, "On the coefficients in the expansion of $e^{e^{t}}$ and $e^{-e^{t}} . "$ Inst. of Actuaries, Jn., v. 76, 1950, p. 152-163.

The expansions mentioned in the title are written

$$
\begin{aligned}
e^{e^{t}} & =e \sum_{n=0}^{\infty} K_{n} t^{n} / n ! \\
e^{-e^{t}} & =e^{-1} \sum_{n=0}^{\infty} A_{n} t^{n} / n !
\end{aligned}
$$

where the $A$ 's and $K$ 's are integers. They are tabulated for $n=0(1) 26$. [See the following review where $K_{n}$ is denoted by $U(n)$ and a table is described for $n=1$ (1)50; the agreement for $n \leqslant 26$ is exact.] The author used the formulas

$$
K_{r}=\sum_{k=1}^{r} \Delta^{k} 0^{r} / k !
$$

and

$$
A_{r}=\sum_{k=1}^{r}(-1)^{k} \Delta^{k} 0^{r} / k !
$$

The values of the Stirling numbers of the second kind, $\Delta^{k} 0^{r} / k$ ! were taken from Fisher \& YATES [as corrected in $M T A C$, v. 4, p. 27] for $r \leqslant 25$. The necessary additional values of these numbers for $r=26$ are appended on p. 163. The table of $A_{r}$ appears to be new. Previous tables of $K_{n}$ for $n \leqslant 20$ are mentioned in FMR, Index $\$ 4.676$.

D. H. L. 